\title{
The Effects of Obstructive Sleep Apnea Syndrome on the Dentate Gyrus and Learning and Memory in Children
}

\author{
비iook Cha, ${ }^{1 *}$ Johanna A. Zea-Hernandez, ${ }^{2 \star}$ Sanghun Sin, ${ }^{2}$ Katharina Graw-Panzer, ${ }^{2}$ Keivan Shifteh, ${ }^{3}$ Carmen R. Isasi, ${ }^{4}$ \\ Mark E. Wagshul, ${ }^{6}$ Eileen E. Moran, ${ }^{5}$ Jonathan Posner, ${ }^{1}$ Molly E. Zimmerman, ${ }^{5}$ and Raanan Arens ${ }^{2}$ \\ ${ }^{1}$ Division of Child and Adolescent Psychiatry, Department of Psychiatry, Columbia University Medical Center, New York State Psychiatric Institute, \\ New York, New York 10032, and 2Division of Respiratory and Sleep Medicine, Children's Hospital at Montefiore, Departments of ${ }^{3}$ Radiology, \\ ${ }^{4}$ Epidemiology and Population Health, and ${ }^{5}$ Neurology, and ${ }^{6}$ Gruss Magnetic Resonance Research Center, Montefiore Medical Center, Albert Einstein \\ College of Medicine, Bronx, New York 10467
}

Obstructive sleep apnea syndrome (OSAS) is associated with intermittent hypoxia and sleep loss. In children, impairments of cognitive function are important manifestations, but the underlying pathology is unknown. We hypothesized that OSAS would affect the dentate gyrus, a hippocampal subdivision essential to neurogenesis and cognition, and that this impact would further affect cognitive function in children. In children with OSAS ( $n=11)$ and control subjects $(n=12$; age and sex matched), we performed diffusion tensor imaging and structural MRI, polysomnography, and neuropsychological assessments. We found that OSAS was associated with decreased mean diffusivity of the left dentate gyrus ( $p=0.002$; false discovery rate corrected; adjusting for sex, age, and body mass index), showing a large effect size (partial $\eta^{2}=0.491$ ), but not with any other structural measures across the brain. Decreased dentate gyrus mean diffusivity correlated with a higher apnea hypopnea index (Spearman's $r=-0.50, p=0.008)$ and a greater arousal index $(r=-0.44, p=0.017)$. OSAS did not significantly affect neuropsychological measures ( $p$ values $>0.5$ ); however, a lower verbal learning score correlated with lower dentate gyrus mean diffusivity $(r=0.54, p=0.004)$. Path analysis demonstrated that dentate gyrus mean diffusivity mediates the impact of OSAS on verbal learning capacity. Finally, the diagnostic accuracy of a regression model based on dentate gyrus mean diffusivity reached $85.8 \%$ (cross validated). This study demonstrates a likely pathway of effects of OSAS on neurocognitive function in children, as well as potential utility of the dentate gyrus mean diffusivity as an early marker of brain pathology in children with OSAS.

Key words: diffusion anisotropy; hippocampus; magnetic resonance imaging

\section{Significance Statement}

In this study we investigate the relationships between dentate gyrus structure, hippocampus-dependent cognition, and obstructive sleep apnea syndrome (OSAS). We demonstrate lower mean diffusivity of the dentate gyrus in children with OSAS, which correlates with a lower verbal learning and memory score. This study provides new evidence of disrupted microstructure of the dentate gyrus in children with OSAS that may help explain some of the neurocognitive deficits described in these children.

\section{Introduction}

Obstructive sleep apnea syndrome (OSAS) in children is a common disorder characterized by recurrent events of partial or complete

\footnotetext{
Received Nov. 15, 2016; revised Feb. 15, 2017; accepted Feb. 17, 2017.

Author contributions: J.A.Z.-H., K.G.-P., K.S., C.R.I., M.E.W., E.E.M., M.E.Z., and R.A. designed research; J.A.Z.-H., S.S., K.G.-P., and M.E.Z. performed research; J.C., S.S., and M.Z. analyzed data; J.C., J.A.Z.-H., J.P., M.Z., and R.A. wrote the paper.

This work was funded by the National Center for Advancing Translational Sciences, a component of the National Institutes of Health, through CTSA (Clinical and Translational Science Awards) Grants UL1TR001073, L2TR001071, and TL1TR001072; National Institute of Mental Health Grant K01-MH109836; and a Brain and Behavior Research Foundation (formally NARSAD) Young Investigator Award.

*J.C. and J.A.Z.-H. contributed equally to this work.

The authors declare no competing financial interests.

Correspondence should be addressed to Dr. Raanan Arens, Division of Respiratory and Sleep Medicine, Children's Hospital at Montefiore, 3415 Bainbridge Avenue, Bronx, NY 10467. E-mail: rarens@montefiore.org.

DOI:10.1523/JNEUROSCI.3583-16.2017

Copyright $\odot 2017$ the authors $\quad 0270-6474 / 17 / 374280-09 \$ 15.00 / 0$
}

airway obstruction leading to perturbations in gas exchange and recurrent arousals (Marcus et al., 2012; Muzumdar and Arens, 2013). The disorder is also prevalent in adults, though the etiology may differ (Arens and Marcus, 2004). However, in both age groups similar consequences are noted (e.g., inflammatory, cardiometabolic derangements, neurocognitive deficits), suggesting common mechanisms leading to organ injury may be similar.

The neurological impairments described in children with OSAS include (1) impairments of behavioral regulation such as aggression, impulsivity, and hyperactivity (Beebe et al., 2004; O'Brien et al., 2004) and (2) impairments in neurocognitive function, particularly in the domains of attention, executive function, motor function, and learning and memory (O'Brien, 2009). However, not all studies support impairments in all neurocognitive domains (Jackman et al., 2012; O'Donoghue et al., 2012). A recent randomized control study known as the Child- 
hood Adenotonsillectomy Trial of children 5-9 years of age with mild to moderate OSAS and mild hypoxemia could not demonstrate impairments in the majority of the neuropsychological tests (Marcus et al., 2013), despite significant altered behavior. In fact, additional evidence of the negative impact of OSAS on neurocognitive function comes from reports demonstrating poor academic achievement in children with mild forms of OSAS and in those with snoring alone (Gozal, 1998; Urschitz et al., 2003; Bourke et al., 2011) .

Intermittent hypoxia has been proposed as a key mechanism leading to the neurocognitive deficits described in children with OSAS (Gozal et al., 2001). Recurrent hypoxic events could lead to loss of neuronal integrity in regions regulating memory and learning (Cervós-Navarro et al., 1991; Bartlett et al., 2004). Additional causes that should be considered and that may affect neurocognition in children are sleep fragmentation and sleep deprivation (Goel et al., 2009; Gevers et al., 2015; Hunter et al., 2016).

Recent neuroimaging studies focusing on the brain and using magnetic resonance imaging (MRI) and magnetic resonance spectroscopic imaging have shown significant changes in gray and white matter in both adults and children with OSAS (Macey et al., 2002; Morrell et al., 2010; Chan et al., 2014; Kumar et al., 2014), particularly in hippocampus and prefrontal cortex (Halbower et al., 2006; Torelli et al., 2011; O'Donoghue et al., 2012), which play important roles in memory, executive function, and motor regulation of breathing, respectively. However, the linkages between these findings, neurocognitive abnormalities, and OSAS remain understudied.

Here, we focus on the role of the hippocampus, particularly the dentate gyrus, in the impact of childhood OSAS on neurocognitive function. The dentate gyrus is one of the few brain regions where neurogenesis occurs, and it plays a key role in learning and memory, emotion, stress response, and spatial navigation (Sahay et al., 2007). Also, the fact that hippocampal neurons are known to be sensitive to oxidative stress (Wang and Michaelis, 2010) may raise an important question about the relationships between the dentate gyrus structure and OSAS. Addressing this may help identify a neural marker for the brain pathology of OSAS.

In this study, we tested the hypothesis that childhood OSAS impacts the structural integrity of the dentate gyrus to a greater extent than that of other brain regions, and that the relationship between OSAS severity measured by either apnea hypopnea index (AHI), oxygen saturation $\left(\mathrm{SpO}_{2}\right)$ nadir, or arousal index (AI) and neurocognitive function is mediated by measures of dentate gyrus integrity. To test this, we investigated the microstructure (diffusion MRI) and macrostructure (structural MRI) of the dentate gyrus using a combination of multimodal MRI and automated hippocampal subdivision segmentation (Iglesias et al., 2015) and assessed learning and memory using neuropsychological testing in children with or without OSAS. Additionally, the region-of-interest investigation was followed by whole-brain analyses of gray and white matter structure using diffusion and structural MRI for completeness of analysis and comparison with the existing literature.

\section{Materials and Methods}

\section{Participants}

All participants were recruited from the population of patients referred for evaluation of sleep disordered breathing at the Sleep Disorders Center at the Children's Hospital at Montefiore (CHAM). The inclusion criterion was age; participants were 13 to 18 years old. Exclusion criteria were preexisting neurological disorders such as seizures, developmental delay, brain tumor, degenerative brain diseases, any craniofacial and genetic syndromes with any CNS involvement, history of head trauma, and cyanotic heart disease or chronic lung disease with hypoxemia; history of vision or hearing impairment or any neurocognitive impairment; contraindications for having an MRI (e.g., claustrophobia, cardiac pacemaker, orthodontics, or having a nonmagnetic resonance compatible surgical/ferromagnetic implant); and being non-English speaking.

The Health Insurance Portability and Accountability Act-compliant study was approved by the Institutional Review Board at Albert Einstein College of Medicine, and informed consent was obtained from each patient and caregiver. We initially enrolled 14 children with OSAS and 13 controls. Of these, 11 OSAS participants (five males and six females) and 12 controls (three males and nine females) completed all aspects of the study (polysomnography, brain MRI, and neuropsychological tests) and were included in final analyses.

\section{Polysomnography}

Participants were studied at the Sleep Disorders Center at the CHAM in a quiet, darkened room. Participants slept under natural sleep conditions. The following parameters were recorded and stored on a computerized polysomnography acquisition and analysis system (XLTEK): chest and abdominal wall movement by respiratory inductance plethysmography (Respitrace Systems; Ambulatory Monitoring), heart rate by ECG, inspired and expired end-tidal $\mathrm{CO}_{2}$ tension $\left(\mathrm{P}_{\mathrm{ET}} \mathrm{CO}_{2}\right)$ by capnography (Capnogard 1265; Novametrix), airflow by nasal pressure (Pro-Tech) and three-pronged thermistor (Nihon Kohden), and $\mathrm{SpO}_{2}$ by pulse oximetry (Masimo). In addition, we obtained the following electroencephalogram derivations (F4-M1, C4-M1, O2-M1, with backup electrodes at F3, C3, O1, and M2), submental and tibial electromyograms, electrooculograms, and continuous infra-red video digital recording.

The sleep stage was performed using standard criteria (Iber et al., 2007). Respiratory events were scored using pediatric standards (Iber et al., 2007). Obstructive apnea was considered as cessation of airflow at the nose and mouth measured by thermal sensor associated with out-ofphase movement of the rib cage and abdomen lasting two breathing cycles. Hypopnea was defined as a decrease of $30 \%$ or more in the amplitude of the nasal pressure transducer associated with a fall in 3\% or more of basal oxygen saturation or with an arousal (Berry et al., 2012). OSAS was determined if the obstructive apnea index was more than 1 episode per hour and/or the AHI was more than five episodes per hour. Hypoventilation was defined as $25 \%$ or more of total sleep time with end-tidal $\mathrm{CO}_{2}>50 \mathrm{mmHg}$

\section{Magnetic resonance imaging acquisition}

Participants were scanned with a 3T Philips scanner at the Gruss Magnetic Resonance Research Center of the Albert Einstein College of Medicine. For structural scans, T1-weighted images were acquired (axial 3D MP-RAGE acquisition; FOV, $240 \mathrm{~mm}$; TE, $4.6 \mathrm{~ms}$; TR, $9.9 \mathrm{~ms}$; voxel size, $1 \times 1 \times 1 \mathrm{~mm}$; sensitivity encoding or SENSE factor, 2.6). We collected diffusion MRI using single-shot spin-echo EPI (TE, $65 \mathrm{~ms}$; TR, 10,000 ms; FOV, $240 \mathrm{~mm}$; voxel size, $2 \times 2 \times 2 \mathrm{~mm}$; noncollinear diffusion sensitizing directions, 32; b, 800 $\mathrm{s} / \mathrm{mm}^{2}$; SENSE acceleration factor, 2.8).

\section{Magnetic resonance imaging analysis}

First, to test our hypothesis on dentate gyrus structural disruption in childhood OSAS, we investigated the microstructure (diffusion MRI) and macrostructure (structural MRI) of the dentate gyrus using automated hippocampal subdivision segmentation. Second, we investigated gray and white matter structure across the whole brain using diffusion and structural MRI for completeness of the study and comparison with the existing literature on effects of OSAS on children's brains. All neuroimaging analyses were conducted on the high-performance computing system at the Department of Systems Biology at Columbia University Medical Center.

Segmentation of hippocampal subdivisions (dentate gyrus). T1-weighted images were processed using an automated parcellation and segmentation method, using the FreeSurfer image analysis suite (http://surfer. nmr.mgh.harvard.edu/; RRID:SCR_001847; Fischl et al., 2002). We then segmented the hippocampal subdivisions, including the dentate gyrus, our structure of interest, using an automated method available in 
FreeSurfer (version 6; RRID:SCR_005996; Iglesias et al., 2015). This method combines a training data set of manual labels based on human ex vivo ultra-high resolution MRI (0.13 $\mathrm{mm}$ isotropic resolution), neuroanatomy of neighboring structures (e.g., amygdala, parahippocampal gyrus) to estimate, and a novel atlas building algorithm based on Bayesian inference to generate more reliable anatomical delineation of the hippocampal subdivisions.

Calculation of diffusion anisotropy in dentate gyrus and other hippocampal subregions. For preprocessing, diffusion-weighted images were skull stripped, eddy-current corrected, and registered to a structural scan in each individual affine registration using FSL's (Functional MRI of the Brain Software Library; RRID:SCR_002823) diffusion toolbox (Jenkinson et al., 2012). Using FSL's DTIFIT program, we calculated the mean diffusivity (MD) and fractional anisotropy (FA) from diffusion-weighted images. To avoid possibilities of suboptimal alignment, we chose not to register the images to a standard space (this was different from our earlier study; Cha et al., 2016). Instead, using a diffusion image registered to a structural image in each participant, we calculated the mean diffusivity of the dentate gyrus and other hippocampal subdivisions. For intrasubject, intermodality registration, we used linear affine registration in FSL [FLIRT (Oxford Centre for Functional MRI of the Brain's Linear Image Registration Tool); Jenkinson et al., 2002].

Voxel-based morphometry. We performed an FSL-VBM (voxel-based morphometry) analysis of the gray matter across the whole brain. We segmented T1-weighted, skull-striped structural scans into gray matter. These gray matter images were then nonlinearly registered using FNIRT (FMRIB's Nonlinear Image Registration Tool) onto a template brain estimated from gray-matter-segmented images across subjects. To avoid bias in template estimation due to the unbalanced numbers of subject across groups, we matched the numbers by randomly choosing 11 out of 12 controls. An estimated gray matter template was used to register the unchosen scan. The resulting registered (warped) gray matter images were smoothed with an isotropic Gaussian kernel with a $\sigma$ of $3 \mathrm{~mm}$.

Tract-based spatial statistics. We performed tract-based spatial statistics (TBSS) in FSL (Smith et al., 2006) to investigate associations of OSAS with white matter integrity (FA) across the whole brain. Following the TBSS pipeline, we performed registration of FA maps to the standard (e.g., Montreal Neurological Institute) space (using a combination of "tbss_2_reg -n" and "tbss_3_postreg -S"), resampling to $1 \mathrm{~mm}$ isotropic voxel space, skeletonization (threshold FA, 0.2), calculation of distance map, and projection of the FA values onto the skeleton.

\section{Neuropsychological evaluation}

Neuropsychological evaluation was performed in all subjects on the day of imaging studies. Premorbid IQ was assessed using the WRAT3 Reading subtest (Wilkinson, 1993). Cognitive assessment was obtained through administration of CogState (Collie et al., 2003; Maruff et al., 2009; www.cogstate.com), a computer-administered battery of tests measuring cognitive function that includes measures of both verbal and visual learning and memory.

Verbal learning and memory were assessed using the International Shopping List Task (ISLT). In the learning condition, the test administrator read the following instructions: "In this task, I am going to read you a shopping list. I would like you to remember as many items from this list as possible. Are you ready to start?" The test administrator then read a list of 16 words to the participant that were presented via the computer screen to the test administrator, but were not within the view of the participant. Words were presented to the participant at a rate of one word per second. A total of three learning trials were presented in this format. Responses were recorded by the test administrator using the computer-based interface. The primary variable of interest for this test was the total number of correct words recalled over three trials. In the delayed recall condition, the test administrator read the following instructions: "Tell me as many of the items from the shopping list as you can remember." This occurred following a delay period of $\sim 15$ min. Responses were again recorded by the test administrator using the computer-based interface.

Visual learning and memory were measured using the Groton Maze Test (GMT). In the learning condition, the participant was presented with a $10 \times 10$ grid of square "tiles" on a computer screen and they were asked to learn a 28-step "pathway" through the maze on the basis of trial and error feedback. Participants were instructed that they could only move one tile at a time; they could only move up, down, left, or right; and they could not move diagonally or backward. In addition, participants were instructed to tap as quickly and as accurately as they could. A total of five learning trials were presented in this format. Responses were recorded using the computer mouse and keyboard. In the delayed recall condition, the test administrator read the following instructions: "You must now find the same hidden pathway that you learned before." The participant was again presented with a $10 \times 10$ grid on the computer screen The participant completed this delayed recall trial once $15 \mathrm{~min}$ after completion of the learning trials. The primary variable of interest for both learning and memory conditions of this task was the number of errors.

\section{Statistical analysis}

Statistical analysis was done using SPSS (RRID:SCR_002865), R (RRID: SCR_001905), and randomize, a permutation test in FSL (for voxelwise analysis; Winkler et al., 2014). Statistical significance was corrected for multiple comparison; e.g., in the dentate gyrus structural measures, since we had no a priori hypothesis about laterality, we first assessed effects of OSAS on two measures (for either MD or volumes) of the hemispheres in separate models and then corrected for two multiple comparisons controlling the false discovery rate (FDR).

Neuropsychological testing. We factored in learning effects during the neuropsychological testing. A recent study with individuals with Alzheimer's disease reported a greater impairment of verbal learning and memory (assessed by ISLT) in a later trial (Thompson et al., 2011). This suggests that learning effects (e.g., sensitization) across multiple trials that are observed in healthy individuals may be disrupted in those with Alzheimer's disease. Based on this a priori knowledge, we factored in potential learning effects by multiple trials: we weighted a score in each trial by multiplying either 1, 2, or 3 (i.e., a trial order) to capture learning effects. We reasoned that this method might maximize any differences in the learning and memory measures between an optimal learner and a suboptimal learner (Thompson et al., 2011). In the case of the GMT, it was not clear whether such learning effects on nonverbal learning and memory could be detected; nevertheless, we tested both unweighted and weighted scores for the GMT as well.

General linear model. To test effects of OSAS on the neural and neuropsycological measures, we used a generalized linear model. The primary outcome variable was either a neural (e.g., dentate gyrus MD or volumes) or neuropsychological measure (ISLT or GMT); the predictor was diagnosis (OSAS/control); and the covariates were age, sex, IQ, body mass index (BMI). Given the group difference in BMI, to avoid any collinearity between OSAS and BMI, we used group-mean-centered BMIs in these models.

Correlation. We used nonparametric methods (Spearman's $\rho$ ) to test correlations of the dentate gyrus MD with clinical and neuropsychological variables among children with OSAS. To determine significance, permutation testing was used; a conditional null distribution was created based on Monte Carlo approximation using the "coin" R package (https://cran.r-project.org/web/packages/coin; Hothorn et al., 2008).

Mediation analysis. We tested the hypothesis that a disrupted dentate gyrus MD mediates the effects of childhood OSAS on verbal learning and memory using mediation analysis ("mediation" R package; Tingley et al., 2014). This method allowed us to assess a confidence interval of the mediation effect itself using rigorous sampling techniques with fewer assumptions of the data. Left dentate gyrus MD was used as the mediator, diagnosis as the treatment variable, weighted ISLT score as the outcome variable, and age, sex, IQ, and BMI as the covariates. The average causal mediation effect (ACME) was determined using a nonparametric bootstrapping method (bias corrected and accelerated; 1000 iterations).

Voxelwise analysis (VBM and TBSS). Our primary interest was testing the impact of OSAS on gray matter volumes and white matter integrity (FA) using VBM and TBSS, respectively. The general linear model containing diagnosis as the regressor and age, sex, BMI, and IQ as the covariates was fitted using randomize in FSL. 
Table 1. Demographic data

\begin{tabular}{lccll}
\hline & OSAS & Control & & \\
& $(n=11)$ & $(n=12)$ & Test statistics & $\begin{array}{l}p \\
\text { value }\end{array}$ \\
\hline Age (years) & $14.8 \pm 1.5$ & $15.1 \pm 1.4$ & Mann-Whitney $U=63$ & 0.88 \\
Height $(\mathrm{cm})$ & $164.3 \pm 8.0$ & $160.1 \pm 6.8$ & Mann-Whitney $U=58$ & 0.62 \\
Weight $(\mathrm{kg})$ & $101.5 \pm 23.9$ & $76.0 \pm 20.8$ & Mann-Whitney $U=24$ & 0.01 \\
BMI $\left(\mathrm{kg} / \mathrm{m}^{2}\right)$ & $38.0 \pm 10.5$ & $29.6 \pm 7.6$ & Mann-Whitney $U=1010.04$ \\
Males $(\%)$ & 45 & 25 & $\chi^{2}=0.44$ & 0.51 \\
Ethnicity & $2 \mathrm{AA}, 9 \mathrm{H}$ & $1 \mathrm{AA}, 1 \mathrm{C}, 1 \mathrm{~A}, 9 \mathrm{H} \chi^{2}=0.95$ & 0.62 \\
Prior adenotonsillectomy & 4 & 1 & $\chi^{2}=1.25$ & 0.26 \\
Handedness & 11 right & 11 right, 1 left & $\chi^{2}=0.96$ & 0.32 \\
IQ & $92.6 \pm 14$ & $98.3 \pm 10.8$ & Mann-Whitney $U=47$ & 0.42 \\
\hline
\end{tabular}

Means \pm SDs and shown. AA, African American; C, Caucasian; A, Asian; H, Hispanic.

As an exploratory analysis, we tested whether a decrease in the dentate gyrus MD was associated with volumetric changes in the neighboring brain regions. The model included the dentate gyrus $\mathrm{MD}$ as the regressor, diagnosis as the regressor of no interest, and the same covariates as in the primary model above. We performed 10,000 Monte Carlo samplingbased random permutations, per a recent report of optimal permutation numbers in a VBM study (Dickie et al., 2015). Multiple comparison correction was done using threshold-free cluster enhancement (Smith and Nichols, 2009).

Receiver-operator characteristic analysis. We performed receiveroperator characteristic (ROC) analysis to test whether hippocampal microstructure measures can reliably predict OSAS using binary logistic regression and leave-one-out cross-validation. Once we obtained diagnostic accuracy (area under curve), we calculated a minimum sample size based on $\alpha$ of 0.05 and $\beta$ of 0.8 using the R package pROC (Robin et al., 2011).

\section{Results \\ Subjects}

Eleven children with OSAS (five males and six females) and 12 controls (three males and nine females) completed polysomnography, neuropsychological tests, and brain MRI and were included in the current analyses. Demographic data are presented in Table 1. No group differences in age, sex, or ethnicity were noted. However, children with OSAS had a higher BMI than the control subjects $(p=0.04)$, though both groups included overweight and obese subjects (three overweight and eight obese children in the OSAS group; three normal, four overweight, and five obese children in the control group). Given the literature on effects of obesity and some of the neural measures (e.g., hippocampal volumes; Hashimoto et al., 2015) that we planned to test in this study, we further adjusted for BMI in all neural and neuropsychological measures in our statistical analyses.

\section{Polysomnography}

Polysomnography data with OSAS participants and controls are presented in Table 2. The OSAS group had moderate to severe OSAS and significant differences compared to the control group in regard to AHI ( $14.8 \pm 7.1$ vs $0.8 \pm 1.1$ events $/ \mathrm{h} ; p<0.001)$, AI $(19.5 \pm 11.0$ vs $6.8 \pm 2.7$ events/h; $p<0.001)$, and $\mathrm{SpO}_{2}$ nadir $(87.4 \pm 11.7$ vs $93.9 \pm 1.4 \% ; p=0.003)$.

\section{Effects of OSAS on hippocampal microstructure}

We found that OSAS was significantly associated with a decrease in left dentate gyrus MD with a large effect size $\left(t=3.92, p_{\mathrm{FDR}}=\right.$ 0.002 , partial $\eta^{2}=0.491$; adjusting for age, sex, IQ, and BMI; no collinearity among the covariates was detected, variance inflation factor of $<1.7$; effects of OSAS were similarly significant without adjusting for BMI, $p=0.001$; Fig. $1 A$ ). In a separate hierarchical
Table 2. Polysomnography

\begin{tabular}{lccll}
\hline & $\begin{array}{l}\text { OSAS } \\
(n=11)\end{array}$ & $\begin{array}{l}\text { Control } \\
(n=12)\end{array}$ & Test statistics & $p$ value \\
\hline AHI (events/h) & $14.8 \pm 7.1$ & $0.8 \pm 1.1$ & Mann-Whitney $U=0$ & $<0.001$ \\
Al (events/h) & $19.5 \pm 11.0$ & $6.8 \pm 2.7$ & Mann-Whitney $U=7$ & $<0.001$ \\
Total sleep time (h) & $5.7 \pm 0.9$ & $5.7 \pm 1.2$ & Mann-Whitney $U=66$ & 0.98 \\
Sleep efficiency (\%) & $80.1 \pm 13.2$ & $81.0 \pm 15.3$ & Mann-Whitney $U=56$ & 0.56 \\
Awakening Index & $6.3 \pm 4.0$ & $3.9 \pm 1.8$ & Mann-Whitney $U=42$ & 0.14 \\
$\quad$ (events/h) & & & & \\
Baseline $\mathrm{SpO}_{2}(\%)$ & $99.7 \pm 0.5$ & $99.4 \pm 0.9$ & Mann-Whitney $U=55$ & 0.52 \\
$\mathrm{SpO}_{2}$ nadir (\%) & $87.4 \pm 11.7$ & $93.9 \pm 1.4$ & Mann-Whitney $U=18$ & 0.003 \\
Baseline ETCO $_{2}$ & $40.4 \pm 3.8$ & $39.5 \pm 4.8$ & Mann-Whitney $U=23$ & 0.81 \\
$\quad(\mathrm{mmHg})$ & & & & \\
Peak ETCO $_{2}$ (mmHg) & $47.6 \pm 7.6$ & $45.2 \pm 4.3$ & Mann-Whitney $U=44$ & 0.28 \\
\hline
\end{tabular}

Means \pm SDs are shown. $\mathrm{ETCO}_{2}$, End-tidal carbon dioxide.

regression, BMI accounted for no significant variance of left dentate gyrus MD without the presence of OSAS $(p=0.118)$, whereas in the presence of OSAS, it accounted for a marginally significant variance $(p=0.054)$. No effects of OSAS were found on the right dentate gyrus $\left(p_{\mathrm{FDR}}=0.501\right)$. Only MD showed the significant effect of OSAS; no significant effects of OSAS were found on volumes or fractional anisotropy of the dentate gyrus in either hemisphere ( $p$ values $>0.12$ ). Dentate gyrus MD averaged across hemispheres showed no significant effects of OSAS $(p=0.5)$.

Across all participants, decreased dentate gyrus MD was significantly correlated with a higher AHI $(r=-0.50, p=0.008$, one-sided Spearman's rank correlation with permutation; Fig. $1 D)$ and with a greater arousal index $(r=-0.44, p=0.017)$. In children with OSAS only, the correlation with arousal index remained significant $(r=-0.57, p=0.033)$, but that with AHI did $\operatorname{not}(r=-0.39, p=0.139)$. No significant correlations of dentate gyrus MD were found with total sleep time, sleep efficiency, awakening index, or $\mathrm{SpO}_{2}$ nadir ( $p$ values $>0.24$ ).

\section{Exploratory analysis in other hippocampal subdivisions.}

Of all the subdivisions of the hippocampus, OSAS was associated with a smaller MD or volume in the CA4, presubiculum, and fimbria with a large effect size (partial $\eta^{2}$ of nearly 0.3 ; Fig. $1 B, C)$.

\section{Effects of childhood OSAS across the whole brain structure}

In whole-brain exploratory analyses, we found no significant impact of OSAS on white matter integrity (measured by FA and MD; TBSS) or gray matter volumes (VBM), with a whole-brain corrected $\alpha$ of 0.05 (see Fig. 3A). No significant results were found in white matter anisotropic measures.

Effects of childhood OSAS on neuropsychological measures Effects of OSAS on ISLT (verbal) and GMT (nonverbal) learning memory did not reach significance (Table 3 ).

Across all participants, lower dentate gyrus MD was correlated with a lower ISLT "learning score" (number of correct responses across learning trials; weighted score, $r=0.54, p=$ 0.004 , one-sided Spearman's rank correlation with permutation; unweighted score, $r=0.42, p=0.022$; Fig. $1 D$ ), but not with ISLT "delayed score" (number of correct responses on a delayed trial; $p=0.36$ ). In children with OSAS only, the correlation with ISLT learning score remained significant $(r=$ $0.59, p=0.028$; unweighted score, $r=0.61, p=0.023$ ). ISLT scores showed no significant correlation with $\mathrm{SpO}_{2}$ nadir or arousal index $(p>0.2)$ either in all participants or in children 
A

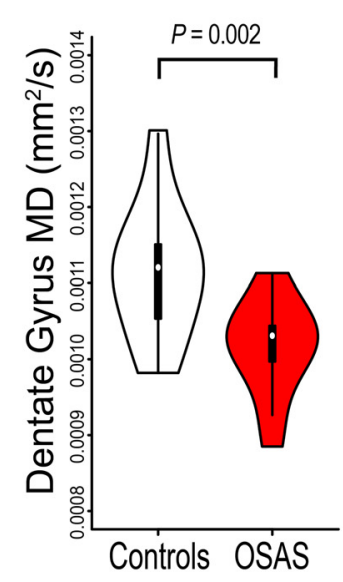

B

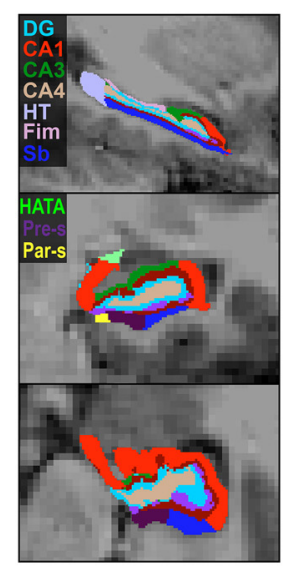

C

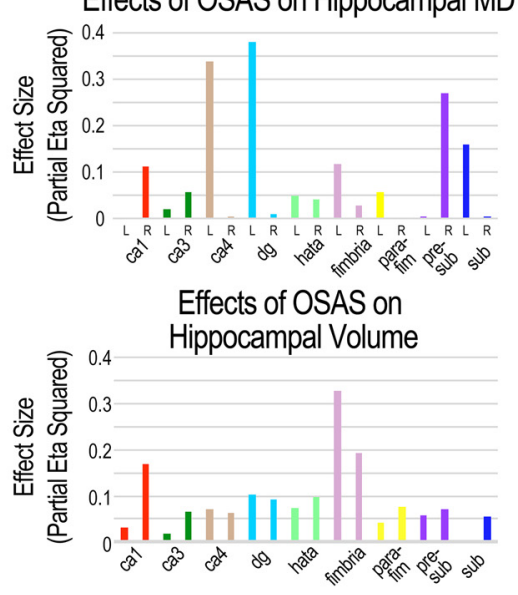

D
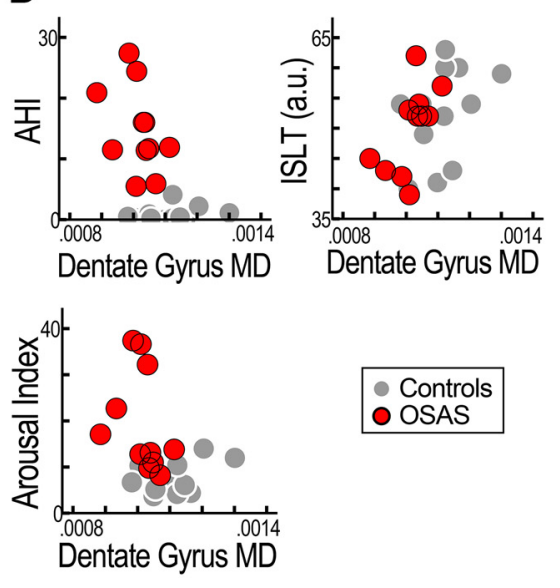

Figure 1. Significant effects of OSAS on dentate gyrus microstructure and cognition in children. $A$, Violin plot of MD of the left dentate gyrus in OSAS subjects and controls (box represents the interquartile range; white dot, median; thin line, $95 \%$ confidence interval; density plot width, frequency). P value indicates significance of the group difference; general linear model; False Discovery Rate controlled. $\boldsymbol{B}$, Representative segmentation of hippocampal subdivisions based on T1-weighted images using an automated segmentation method. $\boldsymbol{C}$, An exploratory analysis showing effects (effect sizes, partial eta squared) of OSAS on hippocampal mean diffusivity and volumes across subdivisions. D, For all subjects (OSAS subjects and controls), a decrease in dentate gyrus mean diffusivity correlates with an increase in apnea hypopnea index, an increase in arousal index, and a decrease in verbal learning (International Shopping List Test). DG, Dentate gyrus; HT, hippocampal tail; Fim, fimbria; HATA, hippocampus, amygdala transition area; Sb, subiculum; Pre-s, presubiculum; Par-s, parasubiculum.

Table 3. Neuropsychological performance

\begin{tabular}{|c|c|c|c|c|}
\hline & $\begin{array}{l}\text { OSAS } \\
(n=11)\end{array}$ & $\begin{array}{l}\text { Control } \\
(n=12)\end{array}$ & $\begin{array}{l}\text { Test } \\
\text { statistic }(F)^{a}\end{array}$ & $p$ value \\
\hline \multicolumn{5}{|l|}{ ISLT learning (No. items correct/16) } \\
\hline Trial 1 & $6.0 \pm 1.3$ & $6.3 \pm 1.4$ & 0.11 & 0.74 \\
\hline Trial 2 & $7.9 \pm 2.0$ & $8.3 \pm 2.1$ & 0.08 & 0.77 \\
\hline Trial 3 & $9.5 \pm 1.4$ & $9.8 \pm 1.3$ & 0.59 & 0.45 \\
\hline Total & $23.3 \pm 3.2$ & $24.4 \pm 3.9$ & 0.001 & 0.93 \\
\hline Weighted ${ }^{b}$ & $50.1 \pm 7.3$ & $52.4 \pm 7.7$ & 0.234 & 0.63 \\
\hline ISLT delay (No. items correct/16) & $9.2 \pm 1.6$ & $8.8 \pm 2.5$ & 1.031 & 0.32 \\
\hline \multicolumn{5}{|l|}{ GMT learning (No. errors) } \\
\hline Trial 1 & $11.8 \pm 4.6$ & $17.5 \pm 4.4$ & 0.36 & 0.55 \\
\hline Trial 2 & $11.6 \pm 2.8$ & $11.3 \pm 4.0$ & 0.05 & 0.82 \\
\hline Trial 3 & $9.1 \pm 3.1$ & $9.5 \pm 4.3$ & 0.09 & 0.76 \\
\hline Trial 4 & $7.2 \pm 4.3$ & $9.2 \pm 5.2$ & 2.50 & 0.13 \\
\hline Trail 5 & $6.4 \pm 2.2$ & $9.4 \pm 7.2$ & 1.43 & 0.24 \\
\hline Total & $51.1 \pm 11.9$ & $56.8 \pm 20.1$ & 1.00 & 0.33 \\
\hline Weighted $^{b}$ & $127.9 \pm 32.4$ & $152.2 \pm 70.4$ & 1.048 & 0.32 \\
\hline GMT delay (No. errors) & $5.5 \pm 4.0$ & $6.9 \pm 4.6$ & 0.77 & 0.39 \\
\hline WRAT3 Reading (standardized score) & $92.6 \pm 14.0$ & $98.3 \pm 10.9$ & 0.23 & 0.63 \\
\hline
\end{tabular}

Means \pm SDs are shown.

${ }^{a}$ Effects of OSAS using a general linear model adjusting for age, sex, BMI (group-mean centered), and IQ.

${ }^{b}$ Weighted by trial order to account for learning effects (Thompson et al., 2011).

with OSAS. No significant correlations of GMT scores (weighted or unweighted) were found with neural or polysomnography measures ( $p$ values $>0.19$ ).

\section{Mediation of dentate gyrus MD between AHI and neuropsychological measures}

We then tested a mechanistic hypothesis that dentate gyrus MD (left) mediates the effect of OSAS on learning and memory capacity. Although no significant direct effects of OSAS on learning and memory was observed, we reasoned this mediation analysis could reveal a plausible causal pathway, otherwise unobservable. We found a significant mediation effect of OSAS on a decrease in verbal memory capacity via a decrease in dentate gyrus MD (ACME, -11.99; $p=0.022$, bias corrected and accelerated; Fig. 2).

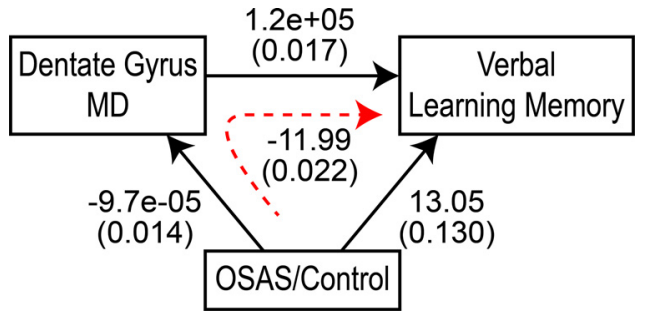

Figure 2. Path analysis shows that decreased left dentate gyrus mean diffusivity mediates the effect of OSAS on learning and memory capacity. Parameter estimates are shown with $p$ values (in brackets). For OSAS subjects/controls, diagnosis was used; for verbal learning and memory, the ISLT was used. The red dashed arrow denotes the ACME. Covariates in the mediation model are not shown (age, sex, and IQ).

\section{Correlation between dentate gyrus MD and volumes across regions}

We tested an exploratory hypothesis that if a decrease in the dentate gyrus MD is related to childhood OSAS, this measure will also correlate with morphometric changes in other regions. We found that a decrease in left dentate gyrus MD significantly correlated with a decrease in gray matter integrity in the left hippocampus, amygdala, thalamus, striatum, and brainstem (whole-brain corrected, $p<0.01$; Fig. $3 B$ ). The regions showing the correlation were subcortical and cortical regions surrounding the hippocampus, such as the parahippocampal gyrus and the ventral visual stream. The correlation was more apparent in the left hemisphere than in the right hemisphere. Of note, in the earlier VBM analysis, no effects of OSAS were shown in those regions.

\section{Prediction of childhood OSAS based on dentate gyrus MD}

Last, we tested whether dentate gyrus MD can reliably predict diagnosis of OSAS in a given individual. Binary logistic regression using dentate gyrus MD showed only modest diagnostic power, with diagnostic accuracy of $69.8 \%$ with sensitivity of $81.8 \%$, specificity of $72.7 \%$, and a diagnostic odds ratio of 12 (leave-one-out cross-validation). A model with dentate gyrus MD and BMI-an important risk factor for OSAS (Peppard et al., 2000)—showed greater diagnostic power with diagnostic accuracy of $85.8 \%$, sen- 

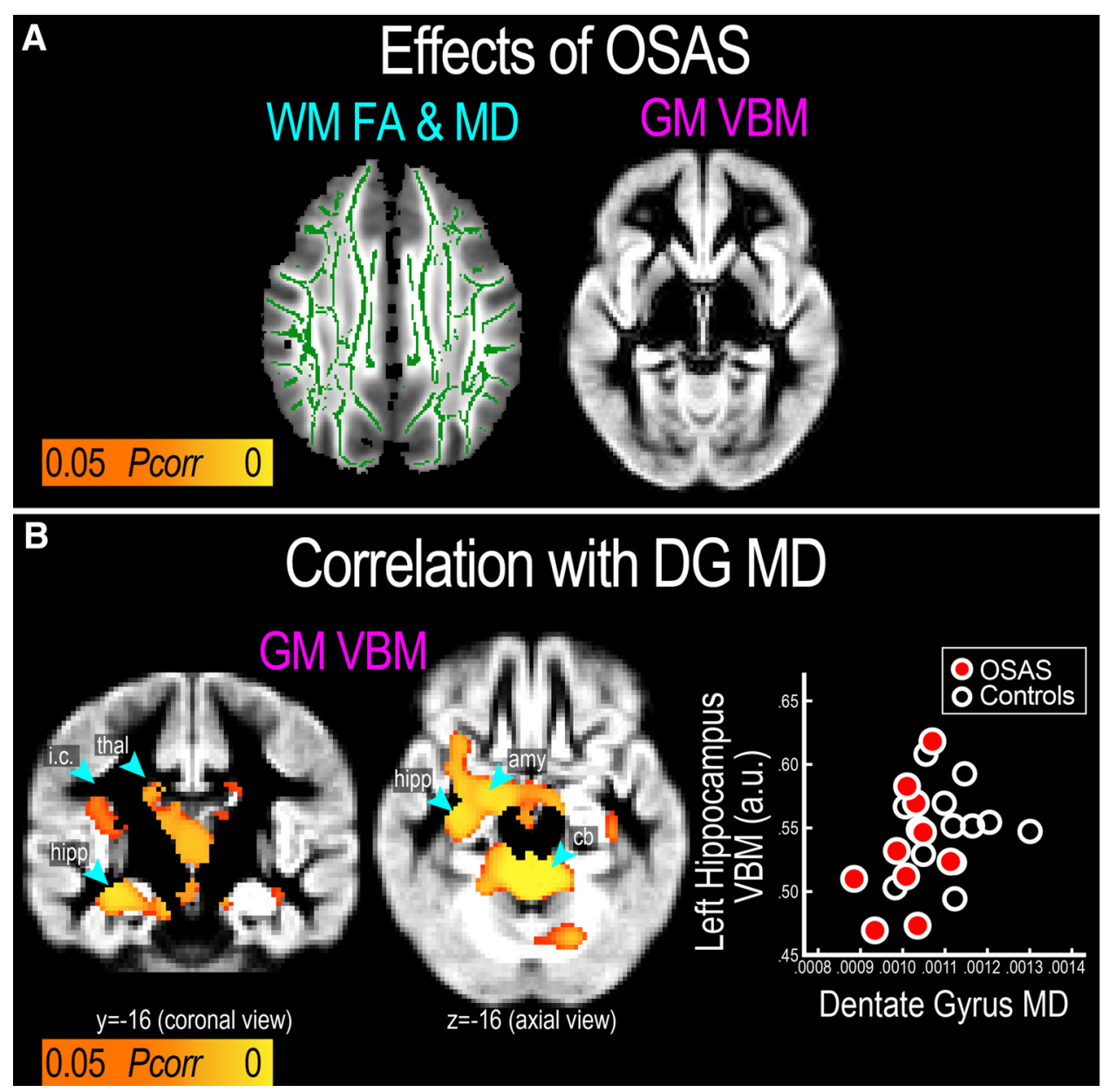

Figure 3. Voxelwise whole-brain analyses on gray and white matter. $A$, No significant group differences were found in fractional anisotropy or mean diffusivity of the white matter (track-based spatial statistics; green represents a group-mean white matter "skeleton" map) or in gray matter intensity (voxel-based morphometry) across the whole brain at corrected $p<0.05 . \boldsymbol{B}$, Voxel-based morphometry analysis shows that a lower dentate gyrus mean diffusivity correlates with a smaller volume in the left hippocampus, amygdala, striatum, and thalamus. The scatter plot is based on relative signal intensity (in arbitrary units) extracted from the ventral hippocampus around the dentate gyrus from VBM analysis. Amy, amygdala; cb, cerebellum; DG, dentate gyrus; GM, gray matter; hipp, hippocampus; i.c., insula cortex; thal, thalamus; WM, white matter.

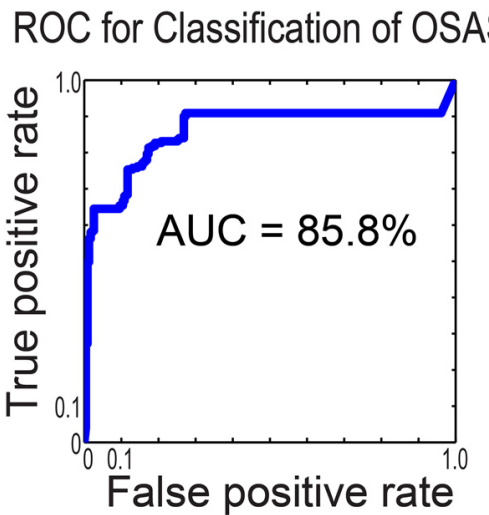

Figure 4. Dentate gyrus mean diffusivity predicts diagnosis of OSAS. An ROC plot shows sensitivity and specificity in the prediction of diagnosis using a logistic regression model with dentate gyrus mean diffusivity and BMI. The ROC analysis was cross-validated (leave-one-out cross-validation). AUC, Area under curve.

sitivity of $90 \%$, specificity of $72.7 \%$, and a diagnostic odds ratio of 24.11. Adding demographic information such as age and sex decreased diagnostic accuracy to 78\% (Fig. 4). Demographic information and BMI without dentate gyrus MD showed diagnostic accuracy of $62.9 \%$. For the model with MD and BMI, a minimum sample size based on an $\alpha$ of 0.05 and $\beta$ of 0.8 was estimated as 9 in each group.

\section{Discussion}

In this study, we found significant associations of childhood OSAS specifically with the microstructure (i.e., mean diffusivity) of the dentate gyrus, but not with the macrostructural measure (i.e., volume) of the dentate gyrus or other brain regions. A decrease in dentate gyrus MD mediated the effect of OSAS on verbal memory capacity and correlated with a decrease in volumes of the neighboring gray matter (such as in the hippocampus and parahippocampal gyrus). This study suggests that disrupted dentate gyrus microstructure may be central to understanding the effects of OSAS on neurocognitive development. The association between OSAS and dentate gyrus microstructure appeared to be independent of BMI. In addition, no significant relationships between BMI and neuropsychological measures were found. Because obesity is an important risk factor for OSAS, it is difficult to disentangle whether the effects are driven largely by obesity, OSAS, or both. Future studies with a larger sample size and a longitudinal design may be able to distinguish between these effects.

Our study extends the literature on the impact of OSAS on neurocognition. Prior human and animal studies demonstrated 
atrophy of widespread brain regions (Macey et al., 2002; Morrell et al., 2003; Huynh et al., 2014), hippocampal damage (Bartlett et al., 2004; Fung et al., 2007), and cognitive deterioration (Décary et al., 2000; Kielb et al., 2012) in OSAS. An early study in children with OSAS (aged 6-16 years) reported a decrease in neuronal metabolites ( $\mathrm{N}$-acetyl aspartate/choline ratio) in the hippocampus and decrements in neuropsychological measures (note that brain-behavioral relationships were not reported in this study; Halbower et al., 2006). Likewise, a decrease in diffusion anisotropic measures in widespread brain structures, including the hippocampus, was reported in a recent study with adults with OSAS (Kumar et al., 2014). In addition to these, the present study also suggests a role of childhood OSAS that may be specific to the dentate gyrus microstructure, with no association with gray matter volumes (VBM) and white matter integrity (TBSS). However, given the small sample size, this study may have been underpowered to detect small effects of OSAS on gray and white matter. The decrease in dentate gyrus MD may indicate an early neural impact of childhood OSAS that may also be associated with subsequent effects on neurocognition. This may be also supported by our exploratory analysis suggesting that the decrease in dentate gyrus MD may mediate the impact of OSAS on verbal learning and memory and the correlation of the decrease in dentate gyrus MD with a decrease in hippocampal/parahippocampal gray matter volumes. However, because of the cross-sectional nature of the study, this mediational analysis needs to be interpreted with caution and confirmed in a larger and prospective study.

What are the neurobiological underpinnings of dentate gyrus diffusivity? Diffusion MRI characterizes microstructural properties of tissues by measuring the displacement of water molecules in the tissues. In an anisotropic structure, such as gray or white matter (compared with an isotropic structure, i.e., CSF), the key factor of degrees of diffusion is permeability of cellular membranes (Assaf and Cohen, 2009). Since anisotropy contrast is poor in the gray matter, mean diffusivity is considered a better metric of gray matter tissue properties than fractional anisotropy. For example, in neurodegenerative disorders such as Alzheimer's disease (Rose et al., 2006) and mild cognitive disorder (Müller et al., 2005; Rose et al., 2006), an increase-rather than a decrease-in hippocampal mean diffusivity correlates with a decrease in hippocampal volume and a cognitive deficit. Although these studies suggest hippocampal diffusivity as a useful metric of pathological microstructural properties, its interpretation requires a careful consideration of the underlying physiology of the hippocampus in the developing brain, for example, neurogenesis. In children's brains, neurogenesis may occur at a higher rate than in adults' brains. (Spalding et al., 2013). Since neurogenesis couples with angiogenesis and vascular remodeling, greater degrees of neurogenesis in children's brains than in adults' brains may reflect a greater magnitude of neurovascular change. Thus, the microstructure of the hippocampus, indexed by mean diffusivity in this study, in children's brains is likely to be different from that in adults' brains; but to our knowledge, this has yet to be tested. This physiological difference with regard to neurogenesis should be considered when interpreting diffusion metrics.

We speculate that the decrease in dentate gyrus MD in childhood OSAS may be related to a change in dentate gyrus microstructure-perhaps resulting from a decrease in hippocampal neurogenesis and the related changes in tissue properties indexed by MD. This may reconcile the results of this study and the literature. First, as learning and memory are often thought to involve dentate gyrus neurogenesis, in our study, decreased dentate gyrus MD in OSAS correlated with a poorer verbal learning score
(ISLT), perhaps indicating altered neurogenesis in the dentate gyrus. This is in line with the recent conceptualization of linking hippocampal neurogenesis to hippocampus-dependent cognition in normal humans (Deng et al., 2010) and in pathological condition such as depression (Sahay et al., 2007). Second, the correlation between a greater OSAS severity (AHI) and smaller dentate gyrus MD may reflect an association between a greater intermittent hypoxia in OSAS and reduced neurogenesis-related microstructural integrity in the dentate gyrus.

Alternative explanations of the decrease in dentate gyrus MD in OSAS merit consideration. Let us consider neurodegeneration. Hypoxia causes neurodegeneration (Nakajima et al., 2000). One might think that intermittent hypoxia in OSAS may initiate an apoptotic process in the hippocampus. This may explain the correlation with a gray matter volume decrease and the mediation effect on cognitive function. Nevertheless, this account directly conflicts with the existing literature reporting a correlation between hippocampal neurodegeneration and an increase, not a decrease, in hippocampal mean diffusivity in older adults (Müller et al., 2005; Rose et al., 2006).

Another consideration may be a compensatory mechanism in response to intermittent hypoxia. Some animal studies report acceleration of neurogenesis in response to intermittent hypoxia (Zhu et al., 2005, 2010). However, it remains to be tested whether the magnitude of intermittent hypoxia used in the laboratory experiments with rodent models is comparable to that of OSAS. Furthermore, the compensatory mechanism does not explain either the correlation with the gray matter volume decrease or the mediation effects on verbal learning and memory. Therefore, neither alternative account may reconcile our data or the literature.

The dentate gyrus MD may confer clinical utility. Our ROC analysis with leave-one-out cross-validation showed the decrease in the dentate gyrus MD along with BMI, a known risk factor of OSAS (Peppard et al., 2000), predicted diagnosis of childhood OSAS with $90 \%$ sensitivity and $72 \%$ specificity. Although it remains to be replicated in a larger sample, this may open the possibility of a neuroimaging-based biomarker of diagnosis or treatment strategies of childhood OSAS. For example, a short $(<15 \mathrm{~min})$ MRI session could offer a quick, reliable, alternative biomarker for the diagnosis of OSAS.

There are limitations worth considering in this study. First, the inferences drawn in this cross-sectional study need to be confirmed with a longitudinal study to formally test the mediation effects of microstructure of the dentate gyrus. Second, potentially suboptimal delineation (particularly for pediatric samples) of the dentate gyrus and the limited spatial resolution of DTI $\left(2 \mathrm{~mm}^{3}\right)$ used in this study could be sources of error (e.g., partial volume effect). Third, we interpret the findings within the OSAS group showing a stronger and statistically significant correlation of dentate gyrus MD with arousals and not with AHI with caution. Based on the moderate association between the dentate gyrus MD and AHI, it is likely that the study may have been underpowered to distinguish whether these effects were driven by arousals alone or also by AHI. Thus, our results remain to be replicated in a larger sample to better refine these effects and the effects of obesity in these children.

\section{References}

Arens R, Marcus CL (2004) Pathophysiology of upper airway obstruction: a developmental perspective. Sleep 27:997-1019. Medline

Assaf Y, Cohen Y (2009) Inferring microstructural information of white matter from diffusion MRI. In: Diffusion MRI: from quantitative measurement to in vivo Neuroanatomy, 2nd ed (Johansen-Berg H, Behrens TEJ, eds), pp 127-146. London: Academic. 
Bartlett DJ, Rae C, Thompson CH, Byth K, Joffe DA, Enright T, Grunstein RR (2004) Hippocampal area metabolites relate to severity and cognitive function in obstructive sleep apnea. Sleep Med 5:593-596. CrossRef Medline

Beebe DW, Wells CT, Jeffries J, Chini B, Kalra M, Amin R (2004) Neuropsychological effects of pediatric obstructive sleep apnea. J Int Neuropsychol Soc 10:962-975. Medline

Berry RB, Budhiraja R, Gottlieb DJ, Gozal D, Iber C, Kapur VK, Marcus CL, Mehra R, Parthasarathy S, Quan SF, Redline S, Strohl KP, Davidson Ward SL, Tangredi MM, American Academy of Sleep Medicine (2012) Rules for scoring respiratory events in sleep: update of the 2007 AASM Manual for the Scoring of Sleep and Associated Events. Deliberations of the Sleep Apnea Definitions Task Force of the American Academy of Sleep Medicine. J Clin Sleep Med 8:597-619. Medline

Bourke R, Anderson V, Yang JS, Jackman AR, Killedar A, Nixon GM, Davey MJ, Walker AM, Trinder J, Horne RS (2011) Cognitive and academic functions are impaired in children with all severities of sleep-disordered breathing. Sleep Med 12:489-496. CrossRef Medline

Cervós-Navarro J, Sampaolo S, Hamdorf G (1991) Brain changes in experimental chronic hypoxia. Exp Pathol 42:205-212. CrossRef Medline

Cha J, Greenberg T, Song I, Blair Simpson H, Posner J, Mujica-Parodi LR (2016) Abnormal hippocampal structure and function in clinical anxiety and comorbid depression. Hippocampus 26:545-553. CrossRef Medline

Chan KC, Shi L, So HK, Wang D, Liew AW, Rasalkar DD, Chu CW, Wing YK, Li AM (2014) Neurocognitive dysfunction and grey matter density deficit in children with obstructive sleep apnoea. Sleep Med 15:1055-1061. CrossRef Medline

Collie A, Maruff P, Makdissi M, McCrory P, McStephen M, Darby D (2003) CogSport: Reliability cognitive tests used in and correlation with conventional postconcussion medical evaluations. Clin J Sport Med 13:28-32. CrossRef Medline

Décary A, Rouleau I, Montplaisir J (2000) Cognitive deficits associated with sleep apnea syndrome: a proposed neuropsychological test battery. Sleep 23:369-381. Medline

Deng W, Aimone JB, Gage FH (2010) New neurons and new memories: how does adult hippocampal neurogenesis affect learning and memory? Nat Rev Neurosci 11:339-350. CrossRef Medline

Dickie DA, Mikhael S, Job DE, Wardlaw JM, Laidlaw DH, Bastin ME (2015) Permutation and parametric tests for effect sizes in voxel-based morphometry of gray matter volume in brain structural MRI. Magn Reson Imaging 33:1299-1305. CrossRef Medline

Fischl B, Salat DH, Busa E, Albert M, Dieterich M, Haselgrove C, van der Kouwe A, Killiany R, Kennedy D, Klaveness S, Montillo A, Makris N, Rosen B, Dale AM (2002) Whole brain segmentation: Automated labeling of neuroanatomical structures in the human brain. Neuron 33:341355. CrossRef Medline

Fung SJ, Xi MC, Zhang JH, Sampogna S, Yamuy J, Morales FR, Chase MH (2007) Apnea promotes glutamate-induced excitotoxicity in hippocampal neurons. Brain Res 1179:42-50. CrossRef Medline

Gevers W, Deliens G, Hoffmann S, Notebaert W, Peigneux P (2015) Sleep deprivation selectively disrupts top-down adaptation to cognitive conflict in the Stroop test. J Sleep Res 24:666-672. CrossRef Medline

Goel N, Rao H, Durmer JS, Dinges DF (2009) Neurocognitive consequences of sleep deprivation. Semin Neurol 29:320-339. CrossRef Medline

Gozal D (1998) Sleep-disordered breathing and school performance in children. Pediatrics 102:616-620. CrossRef Medline

Gozal D, Daniel JM, Dohanich GP (2001) Behavioral and anatomical correlates of chronic episodic hypoxia during sleep in the rat. J Neurosci 21:2442-2450. Medline

Halbower AC, Degaonkar M, Barker PB, Earley CJ, Marcus CL, Smith PL, Prahme MC, Mahone EM (2006) Childhood obstructive sleep apnea associates with neuropsychological deficits and neuronal brain injury. PLoS Med 3:e301. CrossRef Medline

Hashimoto T, Takeuchi H, Taki Y, Yokota S, Hashizume H, Asano K, Asano M, Sassa Y, Nouchi R, Kawashima R (2015) Increased posterior hippocampal volumes in children with lower increase in body mass index: a 3-year longitudinal MRI study. Dev Neurosci 37:153-160. CrossRef Medline

Hothorn T, Hornik K, van de Wiel MA, Zeileis A (2008) Implementing a class of permutation tests: the coin package. J Stat Softw 28:1-23. CrossRef

Hunter SJ, Gozal D, Smith DL, Philby MF, Kaylegian J, Kheirandish-Gozal L (2016) Effect of sleep-disordered breathing severity on cognitive perfor- mance measures in a large community cohort of young school-aged children. Am J Respir Crit Care Med 194:739-747. CrossRef Medline

Huynh NT, Prilipko O, Kushida CA, Guilleminault C (2014) Volumetric brain morphometry changes in patients with obstructive sleep apnea syndrome: effects of CPAP treatment and literature review. Front Neurol 5:58. Medline

Iber C, Ancoli-Israel S, Chesson A, SF Q (2007) The AASM manual for the scoring of sleep and associated events: rules, terminology and technical specifications. Westchester, IL: American Academy of Sleep Medicine.

Iglesias JE, Augustinack JC, Nguyen K, Player CM, Player A, Wright M, Roy N, Frosch MP, McKee AC, Wald LL, Fischl B, Van Leemput K, Alzheimer's Disease Neuroimaging Initiative (2015) A computational atlas of the hippocampal formation using ex vivo, ultra-high resolution MRI: application to adaptive segmentation of in vivo MRI. Neuroimage 115: 117-137. CrossRef Medline

Jackman AR, Biggs SN, Walter LM, Embuldeniya US, Davey MJ, Nixon GM, Anderson V, Trinder J, Horne RS (2012) Sleep-disordered breathing in preschool children is associated with behavioral, but not cognitive, impairments. Sleep Med 13:621-631. CrossRef Medline

Jenkinson M, Bannister P, Brady M, Smith S (2002) Improved optimization for the robust and accurate linear registration and motion correction of brain images. Neuroimage 17:825-841. CrossRef Medline

Jenkinson M, Beckmann CF, Behrens TE, Woolrich MW, Smith SM (2012) FSL. Neuroimage 62:782-790. CrossRef Medline

Kielb SA, Ancoli-Israel S, Rebok GW, Spira AP (2012) Cognition in obstructive sleep apnea-hypopnea syndrome (OSAS): current clinical knowledge and the impact of treatment. Neuromolecular Med 14:180193. CrossRef Medline

Kumar R, Pham TT, Macey PM, Woo MA, Yan-Go FL, Harper RM (2014) Abnormal myelin and axonal integrity in recently diagnosed patients with obstructive sleep apnea. Sleep 37:723-732. Medline

Macey PM, Henderson LA, Macey KE, Alger JR, Frysinger RC, Woo MA, Harper RK, Yan-Go FL, Harper RM (2002) Brain morphology associated with obstructive sleep apnea. Am J Respir Crit Care Med 166:13821387. CrossRef Medline

Marcus CL, Brooks LJ, Draper KA, Gozal D, Halbower AC, Jones J, Schechter MS, Sheldon SH, Spruyt K, Ward SD, Lehmann C, Shiffman RN, American Academy of Pediatrics (2012) Diagnosis and management of childhood obstructive sleep apnea syndrome. Pediatrics 130:576-584. CrossRef Medline

Marcus CL, Moore RH, Rosen CL, Giordani B, Garetz SL, Taylor HG, Mitchell RB, Amin R, Katz ES, Arens R, Paruthi S, Muzumdar H, Gozal D, Thomas NH, Ware J, Beebe D, Snyder K, Elden L, Sprecher RC, Willging P, et al. (2013) A randomized trial of adenotonsillectomy for childhood sleep apnea. N Engl J Med 368:2366-2376. CrossRef Medline

Maruff P, Thomas E, Cysique L, Brew B, Collie A, Snyder P, Pietrzak RH (2009) Validity of the CogState brief battery: relationship to standardized tests and sensitivity to cognitive impairment in mild traumatic brain injury, schizophrenia, and AIDS dementia complex. Arch Clin Neuropsych 24:165-178. CrossRef

Morrell MJ, McRobbie DW, Quest RA, Cummin AR, Ghiassi R, Corfield DR (2003) Changes in brain morphology associated with obstructive sleep apnea. Sleep Med 4:451-454. CrossRef Medline

Morrell MJ, Jackson ML, Twigg GL, Ghiassi R, McRobbie DW, Quest RA, Pardoe H, Pell GS, Abbott DF, Rochford PD, Jackson GD, Pierce RJ, O'Donoghue FJ, Corfield DR (2010) Changes in brain morphology in patients with obstructive sleep apnoea. Thorax 65:908-914. CrossRef Medline

Müller MJ, Greverus D, Dellani PR, Weibrich C, Wille PR, Scheurich A, Stoeter P, Fellgiebel A (2005) Functional implications of hippocampal volume and diffusivity in mild cognitive impairment. Neuroimage 28: 1033-1042. CrossRef Medline

Muzumdar H, Arens R (2013) Physiological effects of obstructive sleep apnea syndrome in childhood. Respir Physiol Neurobiol 188:370-382. CrossRef Medline

Nakajima W, Ishida A, Lange MS, Gabrielson KL, Wilson MA, Martin LJ, Blue ME, Johnston MV (2000) Apoptosis has a prolonged role in the neurodegeneration after hypoxic ischemia in the newborn rat. J Neurosci 20:7994-8004. Medline

O'Brien LM (2009) The neurocognitive effects of sleep disruption in children and adolescents. Child Adolesc Psychiatr Clin N Am 18:813-823. CrossRef Medline 
O’Brien LM, Mervis CB, Holbrook CR, Bruner JL, Klaus CJ, Rutherford J, Raffield TJ, Gozal D (2004) Neurobehavioral implications of habitual snoring in children. Pediatrics 114:44-49. CrossRef Medline

O'Donoghue FJ, Wellard RM, Rochford PD, Dawson A, Barnes M, Ruehland WR, Jackson ML, Howard ME, Pierce RJ, Jackson GD (2012) Magnetic resonance spectroscopy and neurocognitive dysfunction in obstructive sleep apnea before and after CPAP treatment. Sleep 35:41-48. CrossRef Medline

Peppard PE, Young T, Palta M, Dempsey J, Skatrud J (2000) Longitudinal study of moderate weight change and sleep-disordered breathing. JAMA 284:3015-3021. CrossRef Medline

Robin X, Turck N, Hainard A, Tiberti N, Lisacek F, Sanchez JC, Müller M (2011) pROC: an open-source package for R and S+ to analyze and compare ROC curves. BMC Bioinformatics 12:77. CrossRef Medline

Rose SE, McMahon KL, Janke AL, O'Dowd B, de Zubicaray G, Strudwick MW, Chalk JB (2006) Diffusion indices on magnetic resonance imaging and neuropsychological performance in amnestic mild cognitive impairment. J Neurol Neurosurg Psychiatry 77:1122-1128. CrossRef Medline

Sahay A, Drew MR, Hen R (2007) Dentate gyrus neurogenesis and depression. Prog Brain Res 163:697-722. CrossRef Medline

Smith SM, Nichols TE (2009) Threshold-free cluster enhancement: addressing problems of smoothing, threshold dependence and localisation in cluster inference. Neuroimage 44:83-98. CrossRef Medline

Smith SM, Jenkinson M, Johansen-Berg H, Rueckert D, Nichols TE, Mackay CE, Watkins KE, Ciccarelli O, Cader MZ, Matthews PM, Behrens TE (2006) Tract-based spatial statistics: voxelwise analysis of multi-subject diffusion data. Neuroimage 31:1487-1505. CrossRef Medline

Spalding KL, Bergmann O, Alkass K, Bernard S, Salehpour M, Huttner HB, Boström E, Westerlund I, Vial C, Buchholz BA, Possnert G, Mash DC, Druid H, Frisén J (2013) Dynamics of hippocampal neurogenesis in adult humans. Cell 153:1219-1227. CrossRef Medline
Thompson TA, Wilson PH, Snyder PJ, Pietrzak RH, Darby D, Maruff P, Buschke H (2011) Sensitivity and test-retest reliability of the international shopping list test in assessing verbal learning and memory in mild Alzheimer's disease. Arch Clin Neuropsychol 26:412-424. CrossRef Medline

Tingley D, Yamamoto T, Hirose K, Keele L, Imai K (2014) mediation: R package for causal mediation analysis. J Stat Softw 59. CrossRef

Torelli F, Moscufo N, Garreffa G, Placidi F, Romigi A, Zannino S, Bozzali M, Fasano F, Giulietti G, Djonlagic I, Malhotra A, Marciani MG, Guttmann CR (2011) Cognitive profile and brain morphological changes in obstructive sleep apnea. Neuroimage 54:787-793. CrossRef Medline

Urschitz MS, Guenther A, Eggebrecht E, Wolff J, Urschitz-Duprat PM, Schlaud M, Poets CF (2003) Snoring, intermittent hypoxia and academic performance in primary school children. Am J Respir Crit Care Med 168:464-468. CrossRef Medline

Wang X, Michaelis EK (2010) Selective neuronal vulnerability to oxidative stress in the brain. Front Aging Neurosci 2:12. Medline

Wilkinson GS (1993) Wide range achievement test: WRAT3. Wilmington, DE: Wide Range.

Winkler AM, Ridgway GR, Webster MA, Smith SM, Nichols TE (2014) Permutation inference for the general linear model. Neuroimage 92:381-397. CrossRef Medline

Zhu LL, Zhao T, Li HS, Zhao H, Wu LY, Ding AS, Fan WH, Fan M (2005) Neurogenesis in the adult rat brain after intermittent hypoxia. Brain Res 1055:1-6. CrossRef Medline

Zhu XH, Yan HC, Zhang J, Qu HD, Qiu XS, Chen L, Li SJ, Cao X, Bean JC, Chen LH, Qin XH, LiuJH, BaiXC, MeiL, Gao TM (2010) Intermittenthypoxia promotes hippocampal neurogenesis and produces antidepressant-like effects in adult rats. J Neurosci 30:12653-12663. CrossRef Medline 